\title{
Distributed LQR Design for Dynamically Decoupled Systems
}

\author{
Francesco Borrelli*, Tamás Keviczky
}

\begin{abstract}
We consider a set of identical decoupled dynamical systems and a control problem where the performance index couples the behavior of the systems. The coupling is described through a communication graph where each system is a node and the control action at each node is only function of its state and the states of its neighbors. A distributed control design method is presented which requires the solution of a single LQR problem. The size of the LQR problem is equal to the maximum vertex degree of the communication graph plus one. The design procedure proposed in this paper illustrates how stability of the large-scale system is related to the robustness of local controllers and the spectrum of a matrix representing the sparsity pattern of the distributed controller design problem.
\end{abstract}

\section{INTRODUCTION}

Decentralized control techniques today can be found in a broad spectrum of applications ranging from robotics and formation flight to civil engineering. Contributions and interest in this field date back to the early results of [1]. Approaches to decentralized control design differ from each other in the assumptions they make on: $(i)$ the kind of interaction between different systems or different components of the same system (dynamics, constraints, objective), (ii) the model of the system (linear, nonlinear, constrained, continuous-time, discrete-time), (iii) the model of information exchange between the systems, (iv) the control design technique used.

In this paper we focus on identical decoupled linear timeinvariant systems. Our interest in decentralized control for such systems arises from the abundance of networks of independently actuated systems and the necessity of avoiding centralized design when this becomes computationally prohibitive. Networks of vehicles in formation, production units in a power plant, cameras at an airport, an array of mechanical actuators for deforming a surface are just a few examples.

In a descriptive way, the problem of distributed control for decoupled systems can be formulated as follows. A dynamical system is composed of (or can be decomposed into) distinct dynamical subsystems that can be independently actuated. The subsystems are dynamically decoupled but have common objectives, which make them interact with each other. Typically the interaction is local, i.e., the goal of a subsystem is a function of only a subset of other subsystems' states. The interaction will be represented by an "interaction

\footnotetext{
* Corresponding author.

F. Borrelli is with the Dipartimento di Ingegneria, Università degli Studi del Sannio, 82100 Benevento, Italy, francesco.borrelli@unisannio.it

T. Keviczky is with Control and Dynamical Systems, California Institute of Technology, Pasadena, CA 91125, USA, tamasecds.caltech.edu
}

graph", where the nodes represent the subsystems and an edge between two nodes denotes a coupling term in the controller associated with the nodes. Also, typically it is assumed that the exchange of information has a special structure, i.e., it is assumed that each subsystem can sense and/or exchange information with only a subset of other subsystems. We will assume that the interaction graph and the information exchange graph coincide. A distributed control scheme consists of distinct controllers, one for each subsystem, where the inputs to each subsystem are computed only based on local information, i.e., on the states of the subsystem and its neighbors.

Over the past few years, there has been a renewal of interest in systems composed of a large number of interacting and cooperating interconnected units [2]-[15]. A short review of the these approaches can be found in [16].

This manuscript proposes a simple distributed controller design approach and focuses on a class of systems, for which existing methods are either not efficient or would not even be directly applicable. Our method applies to large-scale systems composed of finite number of identical subsystems where the interconnection structure or sparsity pattern is not required to have any special invariance properties. The philosophy of our approach builds on the recent works [9] [11], where at each node, the model of its neighbors are used to predict their behavior. We show that in absence of state and input constraints, and for identical linear system dynamics, such an approach leads to an extremely powerful result: the synthesis of stabilizing distributed control laws can be obtained by using a simple local LQR design, whose size is limited by the maximum vertex degree of the interconnection graph plus one. Furthermore, the design procedure proposed in this paper illustrates how stability of the overall large-scale system is related to the robustness of local controllers and the spectrum of a matrix representing the desired sparsity pattern. In addition, the constructed distributed controller is stabilizing independent of the tuning parameters in the local LQR cost function. This leads to a method for designing distributed controllers for a finite number of dynamically decoupled systems, where the local tuning parameters can be chosen to obtain a desirable global performance. Such result can be immediately used to improve current stability analysis and controller synthesis in the field of decentralized receding horizon control for dynamically decoupled systems [3], [9], [12], [13], [17].

\section{Notation AND PRELIMINARIES}

We denote by $\mathbb{R}$ the field of real numbers, $\mathbb{C}$ the field of complex numbers and $\mathbb{R}^{m \times n}$ the set of $m \times n$ real matrices. 
$\mathbb{C}_{--}=\{s \in \mathbb{C}: \operatorname{Re}(s)<0\} . \mathbb{C}_{-}=\{s \in \mathbb{C}: \operatorname{Re}(s) \leq 0\}$

Notation 1: Let $M \in \mathbb{R}^{m \times n}$, then $M[i: j, k: l]$ denotes a matrix of dimension $(j-i+1) \times(l-k+1)$ obtained by extracting rows $i$ to $j$ and columns $k$ to $l$ from the matrix $M$, with $m \geq j \geq i \geq 1, n \geq k \geq l \geq 1$.

Notation 2: $I_{m}$ denotes the identity matrix of dimension $m, I_{m} \in \mathbb{R}^{m \times m}$.

Notation 3: Let $\lambda_{i}(M)$ denote the $i$-th eigenvalue of $M \in$ $\mathbb{R}^{n \times n}, i=1, \ldots, n$. The spectrum of $M$ will be denoted by $\mathcal{S}(M)=\left\{\lambda_{1}(M), \ldots, \lambda_{n}(M)\right\}$.

Definition 1: A matrix $M \in \mathbb{R}^{n \times n}$ is called Hurwitz (or stable) if all its eigenvalues have negative real part, i.e. $\lambda_{i}(M) \in \mathbb{C}_{--}, i=1, \ldots, n$.

Notation 4: Let $A \in \mathbb{R}^{m \times n}, B \in \mathbb{R}^{p \times q}$. Then $A \otimes B$ denotes the Kronecker product of $A$ and $B$ :

$$
A \otimes B=\left[\begin{array}{ccc}
a_{11} B & \cdots & a_{1 n} B \\
\vdots & \ddots & \vdots \\
a_{m 1} B & \cdots & a_{m n} B
\end{array}\right] \in \mathbb{R}^{m p \times n q} .
$$

Proposition 1: Consider two matrices $A=\alpha I_{n}$ and $B \in$ $\mathbb{R}^{n \times n}$. Then $\lambda_{i}(A+B)=\alpha+\lambda_{i}(B), i=1, \ldots, n$.

Proof: Take any eigenvalue $\lambda_{i}(B)$ and the corresponding eigenvector $v_{i} \in \mathbb{C}^{n}$. Then $(A+B) v_{i}=$ $A v_{i}+B v_{i}=\alpha v_{i}+\lambda_{i} v_{i}=\left(\alpha+\lambda_{i}\right) v_{i}$.

Proposition 2: Given $A, C \in \mathbb{R}^{m \times m}$ and $B \in \mathbb{R}^{n \times n}$, consider two matrices $\bar{A}=\alpha I_{n} \otimes A$ and $\bar{C}=B \otimes C$, where $\bar{A}, \bar{C} \in \mathbb{R}^{n m \times n m}$. Then $\mathcal{S}(\bar{A}+\bar{C})=\bigcup_{i=1}^{n} \mathcal{S}\left(A+\lambda_{i}(B) C\right)$, where $\lambda_{i}(B)$ is the $i$-th eigenvalue of $B$.

Proof: Let $v \in \mathbb{C}^{n}$ be an eigenvector of $B$ corresponding to $\lambda(B)$, and $u \in \mathbb{C}^{m}$ be an eigenvector of $M=(A+\lambda(B) C)$ with $\lambda(M)$ as the associated eigenvalue. Consider the vector $v \otimes u \in \mathbb{C}^{n m}$. Then $(\bar{A}+\bar{C})(v \otimes u)=v \otimes$ $A u+B v \otimes C u=v \otimes A u+\lambda(B) v \otimes C u=v \otimes(A u+\lambda(B) C u)$. Since $(A+\lambda(B) C) u=\lambda(M) u$, we get $(\bar{A}+\bar{C})(v \otimes u)=$ $\lambda(M)(v \otimes u)$.

\section{LQR PROPERTIES FOR DYNAMICALly DeCOUPLED SYSTEMS}

Consider a set of $N_{L}$ identical, decoupled linear timeinvariant dynamical systems, the $i$-th system being described by the continuous-time state equation:

$$
\dot{x}_{i}=A x_{i}+B u_{i}, \quad x_{i}(0)=x_{i 0} .
$$

where $x_{i}(t) \in \mathbb{R}^{n}, u_{i}(t) \in \mathbb{R}^{m}$ are states and inputs of the $i$-th system at time $t$, respectively. Let $\tilde{x}(t) \in \mathbb{R}^{n N_{L}}$ and $\tilde{u}(t) \in \mathbb{R}^{m N_{L}}$ be the vectors which collect the states and inputs of the $N_{L}$ systems at time $t$ :

$$
\begin{aligned}
& \dot{\tilde{x}}=\tilde{A} \tilde{x}+\tilde{B} \tilde{u}, \\
& \tilde{x}(0)=\tilde{x}_{0} \triangleq\left[x_{10}, \ldots, x_{N_{L} 0}\right]^{\prime},
\end{aligned}
$$

with

$$
\tilde{A}=I_{N_{L}} \otimes A, \tilde{B}=I_{N_{L}} \otimes B .
$$

We consider an LQR control problem for the set of $N_{L}$ systems where the cost function couples the dynamic behavior of individual systems:

$$
\begin{aligned}
& J\left(\tilde{u}, \tilde{x}_{0}\right)=\int_{0}^{\infty} \sum_{i=1}^{N_{L}} x_{i}(\tau)^{\prime} Q_{i i} x_{i}(\tau)+u_{i}(\tau)^{\prime} R_{i i} u_{i}(\tau)+ \\
& \left.+\sum_{i=1}^{N_{L}} \sum_{j \neq i}^{N_{L}} x_{i}(\tau)-x_{j}(\tau)\right)^{\prime} Q_{i j}\left(x_{i}(\tau)-x_{j}(\tau) d \tau\right.
\end{aligned}
$$

with

$$
\begin{gathered}
R_{i i}=R_{i i}^{\prime}=R>0, Q_{i i}=Q_{i i}^{\prime}=Q \geq 0 \quad \forall i \\
Q_{i j}=Q_{i j}^{\prime}=Q_{j i} \geq 0 \quad \forall i \neq j .
\end{gathered}
$$

The cost function (5) contains terms which weigh the $i$-th system states and inputs, as well as the difference between the $i$-th and the $j$-th system states and can be rewritten using the following compact notation:

$$
J\left(\tilde{u}(t), \tilde{x}_{0}\right)=\int_{0}^{\infty} \tilde{x}(\tau)^{\prime} \tilde{Q} \tilde{x}(\tau)+\tilde{u}(\tau) \tilde{R} \tilde{u}(\tau) d \tau,
$$

where the matrices $\tilde{Q}$ and $\tilde{R}$ have a special structure defined next. $\tilde{Q}$ and $\tilde{R}$ can be decomposed into $N_{L}^{2}$ blocks of dimension $n \times n$ and $m \times m$ respectively:

$$
\tilde{Q}=\left[\begin{array}{cccc}
\tilde{Q}_{11} & \tilde{Q}_{12} & \cdots & \tilde{Q}_{1 N_{L}} \\
\vdots & \ddots & \vdots & \vdots \\
\tilde{Q}_{N_{L} 1} & \cdots & \cdots & \tilde{Q}_{N_{L} N_{L}}
\end{array}\right], \tilde{R}=I_{N_{L}} \otimes R
$$

with

$$
\begin{aligned}
& \tilde{Q}_{i i}=Q+\sum_{k=1, k \neq i}^{N_{L}} Q_{i k}, \quad i=1, \ldots, N_{L} . \\
& \tilde{Q}_{i j}=-Q_{i j}, \quad i, j=1, \ldots, N_{L}, \quad i \neq j .
\end{aligned}
$$

Remark 1: The cost function structure (5) can be used to describe several practical applications including formation flight, paper machine control and monitoring networks of cameras [14], [18].

Let $\tilde{K}$ and $\tilde{x}_{0}^{\prime} \tilde{P} \tilde{x}_{0}$ be the optimal controller and the value function corresponding to the following LQR problem:

$$
\begin{aligned}
\min _{\tilde{u}} & J\left(\tilde{u}, \tilde{x}_{0}\right) \\
\text { subj. to } & \dot{\tilde{x}}=\tilde{A} \tilde{x}+\tilde{B} \tilde{u} \\
& \tilde{x}(0)=\tilde{x}_{0}
\end{aligned}
$$

Throughout the paper we will assume that a stabilizing solution to the LQR problem (10) with finite performance index exists and is unique (see [19], p. 52 and references therein):

Assumption 1: System $\tilde{A}, \tilde{B}$ is stabilizable and system $\tilde{A}, \tilde{C}$ is observable, where $\tilde{C}$ is any matrix such that $\tilde{C}^{\prime} \tilde{C}=$ $\tilde{Q}$.

We will also assume local stabilizablity and observability:

Assumption 2: System $A, B$ is stabilizable and systems $A, C$ are observable, where $C$ is any matrix such that $C^{\prime} C=$ $Q$.

It is well known that $\tilde{K}=-\tilde{R}^{-1} \tilde{B}^{\prime} \tilde{P}$, where $\tilde{P}$ is the symmetric positive definite solution to the following ARE:

$$
\tilde{A}^{\prime} \tilde{P}+\tilde{P} \tilde{A}-\tilde{P} \tilde{B} \tilde{R}^{-1} \tilde{B}^{\prime} \tilde{P}+\tilde{Q}=0
$$


We decompose $\tilde{K}$ and $\tilde{P}$ into $N_{L}^{2}$ blocks of dimension $m \times n$ and $n \times n$, respectively. Denote by $\tilde{K}_{i j}$ and $\tilde{P}_{i j}$ the $(i, j)$ block of the matrix $\tilde{K}$ and $\tilde{P}$, respectively. In the following theorems we show that $\tilde{K}_{i j}$ and $\tilde{P}_{i j}$ satisfy certain properties which will be critical for the design of stabilizing distributed controllers in Section IV. These properties stem from the special structure of the LQR problem (10). Next, the matrix $X$ is defined as $X=B R^{-1} B^{\prime}$.

Theorem 1: Let $\tilde{K}$ and $\tilde{x}_{0}^{\prime} \tilde{P} \tilde{x}_{0}$ be the optimal controller and the value function solution to the LQR problem (10). Let $\tilde{K}_{i j}=\tilde{K}[(i-1) m: i m,(j-1) n: j n]$ and $\tilde{P}_{i j}=\tilde{P}[(i-1) n:$ $i n,(j-1) n: j n]$ with $i=1, \ldots, N_{L}, j=1, \ldots, N_{L}$.

Then,

1) $\sum_{j=1}^{N_{L}} \tilde{P}_{i j}=P$ for all $i=1, \ldots, N_{L}$, where $P$ is the symmetric positive definite solution of the ARE associated with a single node local problem:

$$
A^{\prime} P+P A-P B R^{-1} B^{\prime} P+Q=0 .
$$

2) $\sum_{j=1}^{N_{L}} \tilde{K}_{i j}=K$ for all $i=1, \ldots, N_{L}$, where $K=$ $-R^{-1} B^{\prime} P$.

Proof: The proof can be found in [16].

Theorem 2: Assume the weighting matrices (9) of the LQR problem (10) are chosen as

$$
\begin{aligned}
& Q_{i i}=Q_{1} \forall i=1, \ldots, N_{L} \\
& Q_{i j}=Q_{2} \forall i, j=1, \ldots, N_{L}, i \neq j .
\end{aligned}
$$

Let $\tilde{x}_{0}^{\prime} \tilde{P} \tilde{x}_{0}$ be the value function of the LQR problem (10) with weights (13), and the blocks of the matrix $\tilde{P}$ be denoted by $\tilde{P}_{i j}=\tilde{P}[(i-1) n: i n,(j-1) n: j n]$ with $i, j=1, \ldots, N_{L}$.

Then $\tilde{P}_{i j}$ is a symmetric negative semidefinite matrix for all $i \neq j$.

Proof: The assumption in (13) requires that the weight $Q_{1}$ used for absolute states and the weight $Q_{2}$ used for neighboring state differences are equal for all nodes and for all neighbors of a node, respectively. Such an assumption and the fact that $\tilde{A}$ and $\tilde{B}$ are block-diagonal with identical blocks, imply that the ARE in (11) is a set of $N_{L}$ identical equations where the matrices $\tilde{P}_{i j}$ are all identical and symmetric for all $i \neq j$. We denote by $\tilde{P}_{2}$, the generic block $\tilde{P}_{i j}$ for $i \neq j$. The ARE equations for the block $\tilde{P}_{i j}$ with $i \neq j$ become

$$
A^{\prime} \tilde{P}_{2}+\tilde{P}_{2} A-\tilde{P}_{2} X \tilde{P}_{1}-\tilde{P}_{1} X \tilde{P}_{2}-\left(N_{L}-2\right) \tilde{P}_{2} X \tilde{P}_{2}-Q_{2}=0,
$$

which can be rewritten as follows in virtue of Theorem 1

$$
(A-X P)^{\prime} \tilde{P}_{2}+\tilde{P}_{2}(A-X P)+\left(N_{L}\right) \tilde{P}_{2} X \tilde{P}_{2}-Q_{2}=0 .
$$

where $P$ is the symmetric positive definite solution of the ARE (12) associated with a single node local problem.

Rewrite equation (15) as

$$
\begin{aligned}
& (A-X P)^{\prime}\left(-N_{L} \tilde{P}_{2}\right)+\left(-N_{L} \tilde{P}_{2}\right)(A-X P)- \\
& +\left(-N_{L} \tilde{P}_{2}\right)^{\prime} X\left(-N_{L} \tilde{P}_{2}\right)+N_{L} Q_{2}=0 .
\end{aligned}
$$

Since $X>0$ and $Q_{2} \geq 0$, equation (16) can be seen as an ARE associated with an LQR problem for the stable system $(A-X P, B)$ with weights $N_{L} Q_{2}$ and $R$. Let the matrix $-N_{L} \tilde{P}_{2}$ be its positive semidefinite solution. Then, the following matrix

$$
\tilde{P}=\left[\begin{array}{cccc}
P-\gamma \tilde{P}_{2} & \tilde{P}_{2} & \ldots & \tilde{P}_{2} \\
\tilde{P}_{2} & P-\gamma \tilde{P}_{2} & \ldots & \tilde{P}_{2} \\
\vdots & \ddots & \ddots & \vdots \\
\tilde{P}_{2} & \ldots & \ldots & P-\gamma \tilde{P}_{2}
\end{array}\right]
$$

with $\gamma=N_{L}-1$ is a symmetric positive definite matrix and it is the unique symmetric positive definite matrix solution to the ARE (11). This proves the theorem.

Under the hypothesis of Theorem 2, because of symmetry and equal weights $Q_{2}$ on the neighboring state differences and equal weights $Q_{1}$ on absolute states, the LQR optimal controller will have the following structure:

$$
\tilde{K}=\left[\begin{array}{cccc}
K_{1} & K_{2} & \cdots & K_{2} \\
K_{2} & K_{1} & \cdots & K_{2} \\
\vdots & \ddots & \ddots & \vdots \\
K_{2} & \cdots & \cdots & K_{1}
\end{array}\right]
$$

with $K_{1}$ and $K_{2}$ functions of $N_{L}, A, B, Q_{1}, Q_{2}$ and $R$.

The following corollaries of Theorem 2 follow from the stability and the robustness of the LQR controller $-R^{-1} B^{\prime}\left(-N_{L} \tilde{P}_{2}\right)$ for system $A-X P$ in (16).

Corollary 1: $A-X P+N_{L} X \tilde{P}_{2}$ is a Hurwitz matrix.

From the gain margin properties [20] we have:

Corollary 2: $A-X P+\alpha N_{L} X \tilde{P}_{2}$ is a Hurwitz matrix for all $\alpha>\frac{1}{2}$, with $\alpha \in \mathbb{R}$.

Remark 2: $A-X P$ is a Hurwitz matrix, thus the system in Corollary 2 is stable for $\alpha=0(A-X P=A+B K$ with $K$ being the $\mathrm{LQR}$ gain for system $(A, B)$ with weights $\left.\left(Q_{1}, R\right)\right)$.

The following condition defines a class of systems and LQR weighting matrices which will be used in later sections to extend the set of stabilizing distributed controller structures.

Condition 1: $A-X P+\alpha N_{L} X \tilde{P}_{2}$ is a Hurwitz matrix for all $\alpha \in\left[0, \frac{1}{2}\right]$, with $\alpha \in \mathbb{R}$.

Essentially, Condition 1 characterizes systems for which the LQR gain stability margin described in Corollary 2 is extended to any positive $\alpha$.

Checking the validity of Condition 1 for a given tuning of $P$ and $\tilde{P}_{2}$ may be performed as a stability test for a simple affine parameter-dependent model

$$
\dot{x}=\underbrace{\left(A_{0}+\alpha A_{1}\right)}_{A(\alpha)} x,
$$

where $A_{0}=A-X P, A_{1}=N_{L} X \tilde{P}_{2}$ and $0 \leq \alpha \leq \frac{1}{2}$. This test can be posed as an LMI problem (Proposition 5.9 in [21]) searching for quadratic parameter-dependent Lyapunov functions.

In the following section we introduce some basic concepts of graph theory before presenting the distributed control design problem. 


\section{LAPLACIAN SPECTRUM OF GRAPHS}

This section is a concise review of the relationship between the eigenvalues of a Laplacian matrix and the topology of the associated graph. We refer the reader to [22], [23] for a comprehensive treatment of the topic. We list a collection of properties associated with undirected graph Laplacians and adjacency matrices, which will be used in subsequent sections of the paper.

A graph $\mathcal{G}$ is defined as

$$
\mathcal{G}=(\mathcal{V}, \mathcal{A})
$$

where $\mathcal{V}$ is the set of nodes (or vertices) $\mathcal{V}=\{1, \ldots, N\}$ and $\mathcal{A} \subseteq \mathcal{V} \times \mathcal{V}$ the set of edges $(i, j)$ with $i \in \mathcal{V}, j \in \mathcal{V}$. The degree $d_{j}$ of a graph vertex $j$ is the number of edges which start from $j$. Let $d_{\max }(\mathcal{G})$ denote the maximum vertex degree of the graph $\mathcal{G}$.

We denote by $\mathbf{A}(\mathcal{G})$ the $(0,1)$ adjacency matrix of the graph $\mathcal{G}$. Let $\mathbf{A}_{i, j} \in \mathbb{R}$ be its $i, j$ element, then $\mathbf{A}_{i, i}=0, \forall i=$ $1, \ldots, N, \mathbf{A}_{i, j}=0$ if $(i, j) \notin \mathcal{A}$ and $\mathbf{A}_{i, j}=1$ if $(i, j) \in \mathcal{A}$, $\forall i, j=1, \ldots, N, i \neq j$.

We will focus on undirected graphs, for which the adjacency matrix $\mathbf{A}(\mathcal{G})$ is symmetric.

Let $\mathcal{S}(\mathbf{A}(\mathcal{G}))=\left\{\lambda_{1}, \ldots, \lambda_{N}\right\}$ be the spectrum of the adjacency matrix $\mathbf{A}$ associated with an undirected graph $\mathcal{G}$ arranged in nondecreasing semi-order.

Property 1: $\lambda_{n} \leq d_{\max }(\mathcal{G})$.

This property together with Proposition 1 implies

Property 2: $\gamma_{i} \geq 0, \forall \gamma_{i} \in \mathcal{S}\left(d_{\max } I_{N}-\mathbf{A}\right)$.

We define the Laplacian matrix of a graph $\mathcal{G}$ in the following way

$$
L(\mathcal{G})=\mathbf{D}(\mathcal{G})-\mathbf{A}(\mathcal{G}),
$$

where $\mathbf{D}(\mathcal{G})$ is the diagonal matrix of vertex degrees $d_{i}$ (also called the valence matrix). Eigenvalues of Laplacian matrices have been widely studied by graph theorists. Their properties are strongly related to the structural properties of their associated graphs. Every Laplacian matrix is a singular matrix. By Geršgorin's theorem [24], the real part of each nonzero eigenvalue of $L(\mathcal{G})$ is strictly positive.

For undirected graphs, $L(\mathcal{G})$ is a symmetric, positive semidefinite matrix, which has only real eigenvalues. Let $\mathcal{S}(L(\mathcal{G}))=\left\{\lambda_{1}, \ldots, \lambda_{N}\right\}$ be the spectrum of the Laplacian matrix $L$ associated with an undirected graph $\mathcal{G}$ arranged in nondecreasing semi-order. Then,

Property 3:

1) $\lambda_{1}=0$ with corresponding eigenvector of all ones, and $\lambda_{2} \neq 0$ iff $\mathcal{G}$ is connected. In fact, the multiplicity of 0 as an eigenvalue of $L(\mathcal{G})$ is equal to the number of connected components of $\mathcal{G}$.

2) The modulus of $\lambda_{i}, i=1, \ldots, N$ is less then $N$.

The second smallest Laplacian eigenvalue $\lambda_{2}$ of graphs is probably the most important information contained in the spectrum of a graph. This eigenvalue, called the algebraic connectivity of the graph, is related to several important graph invariants, and it has been extensively investigated.
Let $L(\mathcal{G})$ be the Laplacian of a graph $\mathcal{G}$ with $N$ vertices and with maximal vertex degree $d_{\max }(\mathcal{G})$. Then properties of $\lambda_{2}(L)$ include

Property 4: $\lambda_{2}(L(\mathcal{G})) \geq 2 \eta(\mathcal{G})\left(1-\cos \frac{\pi}{N}\right)$, where $\eta(\mathcal{G})$ is the edge connectivity of the graph $\mathcal{G}$ [25].

Further relationships between the graph topology and Laplacian eigenvalue locations are discussed in [23] for undirected graphs. Spectral characterization of Laplacian matrices for directed graphs can be found in [24].

\section{Distributed Control Design}

We consider a set of $N$ linear, identical and decoupled dynamical systems, described by the continuous-time timeinvariant state equation (2), rewritten below

$$
\begin{aligned}
& \dot{x}_{i}=A x_{i}+B u_{i}, \\
& x_{i}(0)=x_{i 0} .
\end{aligned}
$$

where $x_{i}(t) \in \mathbb{R}^{n}, u_{i}(t) \in \mathbb{R}^{m}$ are states and inputs of the $i$-th system at time $t$, respectively. Let $\hat{x}(t) \in \mathbb{R}^{N n}$ and $\hat{u}(t) \in \mathbb{R}^{N m}$ be the vectors which collect the states and inputs of the $N$ systems at time $t$, then

$$
\begin{aligned}
& \dot{\hat{x}}=\hat{A} \hat{x}+\hat{B} \hat{u}, \\
& \hat{x}(0)=\hat{x}_{0} \triangleq\left[x_{10}, \ldots, x_{N 0}\right]^{\prime},
\end{aligned}
$$

with

$$
\hat{A}=I_{N} \otimes A, \hat{B}=I_{N} \otimes B .
$$

Remark 3: Systems (22) and (3) differ only in the number of subsystems. We will use system (3) with $N_{L}$ subsystems when referring to local problems, and system (22) with $N$ subsystems when referring to the global problem. Accordingly, tilded matrices will refer to local problems and hatted matrices will refer to the global problem.

We use a graph topology to represent the coupling in the control objective and the communication in the following way. We associate the $i$-th system with the $i$-th node of a graph $\mathcal{G}=(\mathcal{V}, \mathcal{A})$. If an edge $(i, j)$ connecting the $i$-th and $j$ th node is present, then 1 ) the $i$-th system has full information about the state of the $j$-th system and, 2) the $i$-th system control law minimizes a weighted distance between the $i$-th and the $j$-th system states.

The class of $\mathcal{K}_{n, m}^{N}(\mathcal{G})$ matrices is defined as follows:

Definition 2: $\mathcal{K}_{n, m}^{N}(\mathcal{G})=\left\{M \in \mathbb{R}^{n N \times m N} \mid M_{i j}=\right.$ $\mathbf{0}$ if $(i, j) \notin \mathcal{A}, M_{i j}=M[(i-1) n: i n,(j-1) m$ : $j m], i, j=1, \ldots, N\}$

The distributed optimal control problem is defined as follows:

$$
\min _{\hat{K}} \hat{J}\left(\hat{u}, \hat{x}_{0}\right)=\int_{0}^{\infty} \hat{x}(\tau)^{\prime} \hat{Q} \hat{x}(\tau)+\hat{u}(\tau) \hat{R} \hat{u}(\tau) d \tau
$$

subj. to $\dot{\hat{x}}=\hat{A} \tilde{x}+\hat{B} \hat{u}, \hat{u}(t)=\hat{K} \hat{x}(t)$

$$
\begin{aligned}
& \hat{K} \in \mathcal{K}_{m, n}^{N}(\mathcal{G}), \\
& \hat{Q} \in \mathcal{K}_{n, n}^{N}(\mathcal{G}), \quad \hat{R} \in \mathcal{K}_{m, m}^{N}(\mathcal{G}),
\end{aligned}
$$

with $\hat{x}(0)=\hat{x}_{0}, \hat{Q}=\hat{Q}^{\prime} \geq 0$ and $\hat{R}=\hat{R}^{\prime}>0$. We also refer to problem (23) without $(23 \mathrm{c})$ as a centralized 
optimal control problem. In general, computing the solution to problem (23) is an NP-hard problem. Next, we propose a suboptimal control design leading to a controller $\hat{K}$ with the following properties:

1) $\hat{K} \in \mathcal{K}_{m, n}^{N}(\mathcal{G})$

2) $\hat{A}+\hat{B} \hat{K}$ is Hurwitz.

3) Simple tuning of absolute and relative state errors and control effort within $\hat{K}$.

Such controller will be referred to as distributed suboptimal controller. The following theorem will be used to propose a distributed suboptimal control design procedure.

Theorem 3: Consider the LQR problem (10) with $N_{L}=$ $d_{\max }(\mathcal{G})+1$ and weights chosen as in (13) and its solution (17), (18).

Let $M \in \mathbb{R}^{N \times N}$ be a symmetric matrix with the following property:

$$
\lambda_{i}(M)>\frac{N_{L}}{2}, \forall \lambda_{i}(M) \in \mathcal{S}(M) /\{0\} .
$$

and construct the feedback controller:

$$
\hat{K}=-I_{N} \otimes R^{-1} B^{\prime} P+M \otimes R^{-1} B^{\prime} P_{2} .
$$

Then, the closed loop system

$$
A_{c l}=I_{N} \otimes A+\left(I_{N} \otimes B\right) \hat{K}
$$

is asymptotically stable.

Proof: Consider the eigenvalues of the closed-loop system $A_{c l}$ :

$$
\mathcal{S}\left(A_{c l}\right)=\mathcal{S}\left(I_{N} \otimes(A-X P)+M \otimes\left(X P_{2}\right)\right)
$$

By Proposition 2:

$$
\begin{aligned}
& \mathcal{S}\left(I_{N} \otimes(A-X P)+M \otimes\left(X P_{2}\right)\right)= \\
& =\bigcup_{i=1}^{N} \mathcal{S}\left(A-X P+\lambda_{i}(M) X P_{2}\right) .
\end{aligned}
$$

We will prove that $\left(A-X P+\lambda_{i}(M) X P_{2}\right)$ is a Hurwitz matrix $\forall i=1, \ldots, N$, and thus prove the theorem. If $\lambda_{i}(M)=0$ then $A-X P+\lambda_{i}(M) X P_{2}$ is Hurwitz based on Remark 2. If $\lambda_{i}(M) \neq 0$, then from Corollary 2 and from condition (25), we conclude that $A-X P+\lambda_{i}(M) X P_{2}$ is Hurwitz. $\square$

Theorem 3 has several main consequences:

1) If $M \in \mathcal{K}_{1,1}^{N}(\mathcal{G})$, then $\hat{K}$ in (26) is an asymptotically stable distributed controller.

2) We can use one local LQR controller to compose distributed stabilizing controllers for a collection of identical dynamically decoupled subsystems.

3) The first two consequences imply that we can not only find a stabilizing distributed controller with a desired sparsity pattern (which is in general a formidable task by itself), but it is enough to solve a low-dimensional problem (characterized by $d_{\max }(\mathcal{G})$ ) compared to the full problem size (23). This attractive feature of our approach relies on the specific problem structure defined in Section II and IV.
4) The eigenvalues of the closed-loop large-scale system $\mathcal{S}\left(A_{c l}\right)$ can be computed through $N$ smaller eigenvalue computations as $\bigcup_{i=1}^{N} \mathcal{S}\left(A-X P+\lambda_{i}(M) X P_{2}\right)$.

5) The result is independent from the local LQR tuning. Thus $Q_{1}, Q_{2}$ and $R$ in (13) can be used in order to influence the compromise between minimization of absolute and relative terms, and the control effort in the global performance.

For the special class of systems defined by Condition 1, the hypothesis of Theorem 3 can be relaxed as follows:

Theorem 4: Consider the LQR problem (10) with $N_{L}=$ $d_{\max }(\mathcal{G})+1$ and weights chosen as in (13) and its solution (17), (18). Assume that Condition 1 holds.

Let $M \in \mathbb{R}^{N \times N}$ be a symmetric matrix with the following property:

$$
\lambda_{i}(M) \geq 0, \forall \lambda_{i} \in \mathcal{S}(M)
$$

Then, the closed loop system (27) is asymptotically stable when $\hat{K}$ is constructed as in (26).

Proof: Notice that if Condition 1 holds, then $\mathcal{S}(A-$ $X P+\lambda_{i} X P_{2}$ ) is Hurwitz for all $\lambda_{i}(M) \geq 0$ (from Corollary 1 and Corollary 2). By Proposition 2

$$
\begin{aligned}
& \mathcal{S}\left(I_{N} \otimes(A-X P)+M \otimes\left(X P_{2}\right)\right)= \\
& =\bigcup_{i=1}^{N} \mathcal{S}\left(A-X P+\lambda_{i}(M) X P_{2}\right),
\end{aligned}
$$

which together with condition (29) proves the theorem.

In the next sections we show how to choose $M$ in Theorem 3 and Theorem 4 in order to construct distributed suboptimal controllers. The matrix $M$ will $(i)$ reflect the structure of the graph $\mathcal{G}$, (ii) satisfy (25) or (29) and (iii) be computed by using the graph adjacency matrix or the Laplacian matrix.

Next we present the distributed control design for a generic graph structure. Illustrative examples for a simple finite string and for a finite square mesh can be found in [16], [26].

\section{A. Arbitrary Graph Structures}

We consider a generic graph $\mathcal{G}$ for $N$ nodes with an associated Laplacian $L(\mathcal{G})$ and maximum vertex degree $d_{\text {max }}$. Let $0=\lambda_{1}(G) \leq \lambda_{2}(G) \ldots \leq \lambda_{N}(G)$ be the eigenvalues of the the Laplacian $L(\mathcal{G})$. In the next Corollaries 3, 4 and 5 we present three ways of choosing $M$ in (26) which lead to distributed suboptimal controllers.

Corollary 3: Compute $M$ in (26) as $M=a L(\mathcal{G})$. If

$$
a>\frac{N_{L}}{\lambda_{2}(\mathcal{G})},
$$

then the closed loop system (27) is asymptotically stable when $\hat{K}$ is constructed as in (26). In addition, if Condition 1 holds, then the closed loop system (27) is asymptotically stable for all $a \geq 0$.

Proof: The proof is a direct consequence of Theorems 3 and 4, and Property 3 of the Laplacian matrix.

Remark 4: By using Property 4, condition (30) can be linked to the edge connectivity as follows

$$
a>\frac{N_{L}}{2 \eta(\mathcal{G}) 1-\cos \frac{\pi}{N}} .
$$


Remark 5: Corollary 3 links the stability of the distributed controller to the size of the second smallest eigenvalues of the graph Laplacian. It is well known that graphs with large $\lambda_{2}$ (with respect to the maximal degree) have some properties which make them very useful in several applications such as computer science. Interestingly enough, this property is shown here to be crucial also for the design of distributed controllers. We refer the reader to [23] for a more detailed discussion on the importance of the second largest eigenvalue of a Laplacian.

Corollary 4: Compute $M$ in (26) as $M=a I_{N}-$ $b \mathbf{A}(\mathcal{G}), b \geq 0$. If $a-b d_{\max }>\frac{N_{L}}{2}$, then the closed loop system (27) is asymptotically stable when $\hat{K}$ is constructed as in (26). In addition, if Condition 1 holds, then the closed loop system (27) is asymptotically stable if $a-b d_{\max } \geq 0$.

Proof: Notice that $\lambda_{\min }(M)=a-b \lambda_{\max }(\mathbf{A}(\mathcal{G})) \geq$ $a-b d_{\max }$. The proof is a direct consequence of Theorems 3 and 4 and Property 1 of the adjacency matrix.

Consider a weighted adjacency matrix $\mathbf{A}^{w}=\mathbf{A}^{w}(\mathcal{G})$, defined as follows. Denote by $\mathbf{A}_{i, j}^{w} \in \mathbb{R}$ its $i, j$ element, then $\mathbf{A}_{i, j}^{w}=0$, if $i=j$ and $(i, j) \notin \mathcal{A}$ and $\mathbf{A}_{i, j}^{w}=w_{i j}$ if $(i, j) \in \mathcal{A}, \forall i, j=1, \ldots, N, i \neq j$. Assume $w_{i j}=w_{j i}>0$. Define $w_{\max }$ as $w_{\max }=\max _{i} \sum_{j} w_{i j}$

Corollary 5: Compute $M$ in (26) as $M=a I_{N}-\mathbf{A}^{w}(\mathcal{G})$. If $a>w_{\max }-\frac{N_{L}}{2}$, then the closed loop system (27) is asymptotically stable when $\hat{K}$ is constructed as in (26). In addition, if Condition 1 holds, then the closed loop system (27) is asymptotically stable if $a \geq w_{\max }$.

Proof: $\mathbf{A}^{w} \mathbf{1} \leq w_{\max } \mathbf{1}$ and by Perron-Frobenius Theorem $\lambda_{\max }\left(\mathbf{A}^{w}\right) \leq w_{\max }$. Notice that $\lambda_{\min }(M)=a-$ $\lambda_{\max }(\mathbf{A}(\mathcal{G})) \geq a-w_{\max }$, then the proof is a direct consequence of Theorems 3 and 4 .

The results of Corollaries 3-5 are summarized in Table I.

\begin{tabular}{|c|c|c|}
\hline Choice of $M$ & S.C. & $\begin{array}{c}\text { S.C. if } \\
\text { Cond. 1 Holds }\end{array}$ \\
\hline \hline$M=a L(\mathcal{G})$ & $a>\frac{N_{L}}{\lambda_{2}(\mathcal{G})}$ & $a \geq 0$ \\
\hline $\begin{array}{c}M=a I_{N}-b \mathbf{A}(\mathcal{G}), \\
b \geq 0\end{array}$ & $a-b d_{\max }>\frac{N_{L}}{2}$ & $a-b d_{\max } \geq 0$ \\
\hline$a I_{N}-\mathbf{A}^{w}(\mathcal{G})$ & $a>w_{\max }-\frac{N_{L}}{2}$ & $a \geq w_{\max }$ \\
\hline
\end{tabular}

TABLE I

SUMMARY OF STABILITY CONDITIONS (S.C.) IN COROLLARIES 3-5 FOR THE CLOSED LOOP SYSTEM (26)-(27).

Corollaries 3-5 present three choices of distributed control design with increasing degrees of freedom. In fact, $a, b$ and $w_{i j}$ are additional parameters which, together with $Q_{1}, Q_{2}$ and $R$, can be used to tune the closed-loop system behavior. We recall here that from Theorem 3, the eigenvalues of the closed-loop large-scale system are related to the eigenvalues of $M$ through the simple relation (28). Thus as long as the stability conditions defined in Table I are satisfied, the overall system architecture can be modified arbitrarily by adding or removing subsystems and interconnection links. This leads to a very powerful modular approach for designing distributed control systems.

\section{REFERENCES}

[1] S. Wang and E. J. Davison, "On the stabilization of decentralized control systems," IEEE Trans. Automatic Control, vol. 18, no. 5, pp. 473-478, 1973.

[2] R. D'Andrea and G. E. Dullerud, "Distributed control design for spatially interconnected systems," IEEE Trans. Automatic Control, vol. 48, no. 9, pp. 1478-1495, Sept. 2003.

[3] E. Camponogara, D. Jia, B. H. Krogh, and S. Talukdar, "Distributed model predictive control," IEEE Control Systems Magazine, Feb. 2002.

[4] J. A. Fax and R. M. Murray, "Graph laplacians and stabilization of vehicle formations," in Proc. 2002 IFAC World Congress, Barcelona, Spain, June 2002.

[5] M. R. Jovanović, "On the optimality of localized distributed controllers," in Proc. American Contr. Conf., June 2005, pp. 4583-4588.

[6] C. Langbort, R. S. Chandra, and R. D'Andrea, "Distributed control design for systems interconnected over an arbitrary graph," IEEE Trans. Automatic Control, vol. 49, no. 9, pp. 1502-1519, Sept. 2004.

[7] V. Gupta, B. Hassibi, and R. M. Murray, "A sub-optimal algorithm to synthesize control laws for a network of dynamic agents," Int. J. Control, vol. 78, no. 16, pp. 1302-1313, Nov. 2005.

[8] M. Rotkowitz and S. Lall, "A characterization of convex problems in decentralized control," IEEE Trans. Automatic Control, vol. 51, no. 2, pp. 274-286, Feb. 2006.

[9] T. Keviczky, F. Borrelli, and G. J. Balas, "A study on decentralized receding horizon control for decoupled systems," in Proc. American Contr. Conf., 2004.

[10] — "Stability analysis of decentralized RHC for decoupled systems," in 44th IEEE Conf. on Decision and Control, and European Control Conf., Seville, Spain, Dec. 2005.

[11] _ _ "Decentralized receding horizon control for large scale dynamically decoupled systems," Automatica, to appear 2006.

[12] W. B. Dunbar and R. M. Murray, "Receding horizon control of multivehicle formations: A distributed implementation," in Proc. 43rd IEEE Conf. on Decision and Control, 2004.

[13] A. Richards and J. How, "A decentralized algorithm for robust constrained model predictive control," in Proc. American Contr. Conf., 2004.

[14] F. Borrelli, T. Keviczky, G. J. Balas, G. Stewart, K. Fregene, and D. Godbole, "Hybrid decentralized control of large scale systems," in Hybrid Systems: Computation and Control, ser. Lecture Notes in Computer Science, vol. 3414. Springer Verlag, Mar. 2005, pp. 168183.

[15] D. Stipanovic, G. Inalhan, R. Teo, and C. J. Tomlin, "Decentralized overlapping control of a formation of unmanned aerial vehicles," Automatica, vol. 40, no. 8, pp. 1285-1296, 2004.

[16] F. Borrelli and T. Keviczky, "Distributed lqr design for identical dynamically decoupled systems," Università del Sannio, Benevento, Italy, Tech. Rep. TR390, Semptember 2006. [Online]. Available: http://www.grace.ing.unisannio.it/publication/390

[17] V. Gupta, B. Hassibi, and R. M. Murray, "On the synthesis of control laws for a network of autonomous agents," in Proc. American Contr. Conf., 2004.

[18] T. Keviczky, F. Borrelli, G. J. Balas, K. Fregene, and D. Godbole, "Decentralized receding horizon control and coordination of autonomous vehicle teams," IEEE Transaction Control System Technology, to appear 2006.

[19] B. D. O. Andreson and J. B. Moore, Optimal Control: Linear Quadratic Methods. Englewood Cliffs, N.J.: Prentice Hall, 1990.

[20] M. G. Safonov and M. Athans, "Gain and phase margin for multiloop LQG regulators," IEEE Trans. Automatic Control, vol. AC-22, no. 2, pp. 173-179, Apr. 1977.

[21] C. Scherer and S. Weiland, Linear Matrix Inequalities in Control, 2000, version 3.0.

[22] R. Merris, "Laplacian matrices of graphs: A survey," Linear Algebra and Its Applications, vol. 197, pp. 143-176, 1994.

[23] B. Mohar, "The laplacian spectrum of graphs," Graph Theory, Combinatorics, and Applications, vol. 2, pp. 871-898, 1991.

[24] R. Agaev and P. Chebotarev, "On the spectra of nonsymmetric laplacian matrices," Linear Algebra and Its Applications, vol. 399, no. 5, pp. 157-168, 2005.

[25] B. Bollobás, Modern Graph Theory. S Springer, 2002.

[26] Online, "www.aem.umn.edu/Kkeviczky/Simulations.html," 2005. 